\title{
Atomically resolved tomographic reconstruction of nanoparticles from Single Projection: influence of amorphous carbon support
}

\section{Pritam Banerjee}

Department of Metallurgical and Materials Engineering, Indian Institute of Technology Madras, Chennai, India - 600036

\section{Chiranjit Roy}

Department of Metallurgical and Materials Engineering, Indian Institute of Technology Madras, Chennai, India - 600036

\section{Subhra Kanti De}

Department of Metallurgical and Materials Engineering, Indian Institute of Technology Madras, Chennai, India - 600036

\section{Antonio J. Santos}

Department of Materials Science and Metallurgic Engineering, and Inorganic Chemistry, Faculty of Sciences, University of Cádiz, Puerto Real, 11510 Cádiz, Spain

\section{Francisco M. Morales}

Department of Materials Science and Metallurgic Engineering, and Inorganic Chemistry, Faculty of Sciences, University of Cádiz, Puerto Real, 11510 Cádiz, Spain

\section{Somnath Bhattacharyya ( $\sim$ somnath.iitm@gmail.com)}

Department of Metallurgical and Materials Engineering, Indian Institute of Technology Madras, Chennai, India -600036

\section{Research Article}

Keywords: Tomographic reconstruction, In-line 3D holography, Channelling theory, nanoparticle, TEM

Posted Date: October 26th, 2020

DOI: https://doi.org/10.21203/rs.3.rs-95300/v1

License: (1) (i) This work is licensed under a Creative Commons Attribution 4.0 International License. Read Full License

Version of Record: A version of this preprint was published at Ultramicroscopy on February 1st, 2021. See the published version at https://doi.org/10.1016/j.ultramic.2020.113177. 


\section{Abstract}

Nanoparticles have a wide range of applications due to their unique geometry and arrangement of atoms. For a precise structure-property correlation, information regarding atomically resolved 3D structures of nanoparticles is utmost beneficial. Though modern aberration-corrected transmission electron microscopes can resolve atoms with sub-angstrom resolution, an atomic-scale 3D reconstruction of nanoparticle is a challenge using tilt series tomography due to high radiation damage. Instead, inline 3D holography based tomographic reconstructions from single projection registered at low electron doses are more suitable for defining atoms dispositions at nanostructures. Nanoparticles are generally supported on amorphous carbon film for TEM experiments. However, neglecting the influence of carbon film on the tomographic reconstruction of the nanoparticle may lead to ambiguity. In order to address this issue, the effect of amorphous carbon support was quantitatively studied using simulations and experiments.

\section{Introduction}

Nanoparticles have immense potential applications in various fields ranging from electronics ${ }^{[1]}$ to renewable energy ${ }^{[2]}$, medicine ${ }^{[3]}$ to agriculture sectors ${ }^{[4]}$ due to their unique physical ${ }^{[5]}$ and chemical properties ${ }^{[6]}$. To study structure-property correlation precisely, atomically resolved 3D structural information of nanoparticle is very crucial ${ }^{[7]}$. Tomographic techniques such as atom probe tomography $(\mathrm{APT})^{[8]}$ and electron tomography ${ }^{[9]}$ are the most popular techniques for 3D reconstruction of nanostructures. The major challenges that limit the use of APT for atomic-scale characterization of nanoparticles are sample preparation technique and escape of atoms during acquisition. The nanoparticles should be embedded in a matrix for APT analysis without residual voids ${ }^{[10],[11]}$. These voids create artefacts during 3D reconstruction, and loss of atoms (around $50 \%$ ) limits the atomic-scale resolution in tomograms ${ }^{[8]}$. On the contrary, the sample preparation technique of nanoparticles for transmission electron microscope (TEM) experiment is straightforward, and pristine structural details can be retrieved using electron tomography [9],[12]. Based on the used modes of operation of TEM, electron tomography can be classified as; (1) Scanning transmission electron microscope (STEM) based and (2) TEM based. STEM tomography reconstructs 3D structure from 2D projected images acquired at different angles by rotating or tilting the specimen ${ }^{[13],[14]}$. For reconstructing atomic resolution tomograms this technique faces several challenges; (1) the 'missing wedge' results in the incomplete acquisition of tilt series images due to limited tilt $\left( \pm 70^{\circ}\right)$ of the sample holders ${ }^{[15]},(2)$ radiation damage of beam sensitive samples limits types of sample that can be examined ${ }^{[16]}$ and (3) it is extremely difficult to align tilt series images exactly to the common axis ${ }^{[17]}$. Hence, TEM based tomographic technique from a single projection at a low electron dose is suitable for tomographic reconstruction of nanoparticles ${ }^{[9],[18]}$. TEM based tomography, also termed as in-line 3D holography ${ }^{[9]}$, determines composition and depth information from the exit wave at the exit surface of the sample. The exit wave comprises of entire field information [19],[20],[21],[22], such as amplitude and phase retrieved from the complex-valued exit wave 
reconstruction using a set of high-resolution focal series images along with a low index zone axis orientation. The column mass and exit surface geometry information are obtained by the electron channelling theory [23],[24],[25] using a reconstructed exit wave. To satisfy electron channelling, the incident electrons should scatter dynamically and channel through the atomic columns without interfering the neighbouring atomic columns. The atoms in the atomic column focus the electron wave, leads to periodic oscillation of wave over a constant thickness, known as extinction distance. To channel incident electrons, the specimen should be thin, and distances between atomic columns orthogonal to the projection direction should be greater than $1 \AA^{[23]}$. Electron channelling theory works well for both periodically crystalline objects as well as for non-periodic molecular structure and defective crystals ${ }^{[26]}$. Till now, simulated structure of gold wedge sample ${ }^{[18]}$, bi-layer graphene and non-periodic oleic acid molecules ${ }^{[26]}$ were reconstructed in $3 \mathrm{D}$ at atomic resolution. Also, atomic resolution tomographic reconstruction of experimental bulk specimens such as gold bridge, $\mathrm{MgO}$ molecule and germanium wedge shaped sample were reported in the literature ${ }^{[9]}$. Nanoparticles are generally supported on commercially available amorphous carbon film deposited on Cu mesh grids. In most of the cases, supporting amorphous carbon films effects are being neglected during interpretation of atomic resolution images of nanoparticles though it was reported recently that carbon support induces lattice strain artefacts within nanoparticles ${ }^{[27]}$. So far, atomic resolution tomograms of $\mathrm{NiO}^{[28]}, \mathrm{CeO}_{2}$ and $\mathrm{MoS}_{2}$ nanoparticles ${ }^{[29]}$ have been presented in conference proceedings but the influence of supporting amorphous carbon films on atomically resolved tomographic reconstruction has not been considered yet which leaves ambiguity in final reconstructed tomogram. The motivation of this present work is to uncover the influence of supporting amorphous carbon films on tomographic reconstructions of nanoparticles quantitatively.

\section{Results And Discussion}

\section{Simulation results:}

Figure 1 portrays the influence of supporting amorphous carbon film on the atomically resolved tomographic reconstruction of simulated disc shape single-crystalline non-defective silver nanoparticle from single projection using in-line 3D holography ${ }^{[9]}$.

Each row of Fig. 1 displays the results related to simulated nanostructures comprising nanoparticle and amorphous carbon support of varying $t_{p} / t_{a c}$ ratio, where $t_{p}$ is the thickness of the nanoparticle and $t_{a c}$ is the thickness of supporting amorphous carbon film. Column 1 and column 2 (from left to right) of Fig. 1 represent the simulated nanostructures comprises nanoparticle supported on amorphous carbon film and the reconstructed phases of the complex valued exit waves of various $t_{p} / t_{a c}$ ratios and density $\left(\rho_{C}=2.1\right.$ $\mathrm{g} / \mathrm{cc}$ and $3 \mathrm{~g} / \mathrm{cc}$ ) along [001] direction respectively. For each nanostructure, one simulated TEM image from the set of through focal series and corresponding amplitude of the reconstructed exit wave is presented in first two columns of Fig. S1. The reconstructed phases are shown in column 2 of Fig. 1 reveal that half of the particle is hanging in a vacuum while the rest is supported on amorphous carbon. It 
is noticeably evident that the atomic columns gradually loose contrast and become blurred with increasing carbon thickness. Complex valued wave is retrieved at the exit surface (exit face) of the amorphous carbon block; hence the atomic columns with increasing carbon thickness become out of focus. As an outcome, the interface between the unsupported and carbon-supported region becomes prominent with increasing carbon thickness.

The image region marked with a yellow rectangle box in row 1, column 2 includes total of 200 atomic columns distributed equally in both unsupported and supported regions. Precisely the same region is maintained for all the simulated nanostructures for tomographic reconstruction ${ }^{[9]}$. To obtain the focus corrected wave (FCW ${ }^{[9]}$, the wave reconstructed at the exit surface is propagated locally. The correct defocus value for each atomic column of the selected region is calculated using maximum propagated intensity (MPI) criteria ${ }^{[18]}$. According to this criteria, the background-subtracted wave is obtained by normalising reconstructed exit wave with valley wave (background). The background-subtracted waves were propagated in a defocus range of -20 to $+20 \AA$ with a defocus step of $1 \AA$ for all cases of this present work. The exact focus for an atomic column was determined by finding the maximum intensity of the propagated wave as a function of defocus. Since the defocus range was different for each carbon thickness, the defocus values were normalised by subtracting the average defocus values of unsupported regions of each atomic column. The intensity profile of two different atomic columns ( $A$ and $B$ type stacking) of the unsupported region is shown in column 3 of Fig. S1 for all the simulated nanostructures.

The FCW plotted in the complex plane, known as Argand plot, provides visual insights regarding the interaction of accelerated electrons with matter ${ }^{[24]}$. Column 3 of Fig. 1 represents the mass circle in Argand plane (Argand plot) and phase angle $\theta^{\prime}$. It is clearly visible that with increasing carbon thickness, the red dots representing the complex-valued FCWs are scattered away from the mass circle. For same $t_{\mathrm{p}} / \mathrm{t}_{\mathrm{ac}}$ ratio, scattering is even higher for higher carbon density as evident from the mass circle of row 6 $\left(\mathrm{t}_{\mathrm{p}} / \mathrm{t}_{\mathrm{ac}}=0.25^{\star}, \rho_{\mathrm{C}}=3 \mathrm{~g} / \mathrm{cc}\right)$

Column 4 of Fig. 1 represents the normalized focus maps that represent the exit surface geometry or surface roughness of simulated Ag disc-shaped nanoparticle. The normalised focus maps indicate that in the absence of carbon, the exit surface exhibits uniform roughness, which proves the proficiency of the tomographic reconstruction procedure adopted in this work. In unsupported regions, two adjacent atomic columns with $A$ and $B$ type stacking exhibit a defocus difference of $2 \AA$ (column 3 of Fig. S1) that is equal to the difference in distance along direction [001] between $A$ and $B$ layer of Ag unit cell (Table 1). The histogram of the normalized defocus values of the unsupported region reveals equal counts for two alternate atomic columns with a difference in focus values of $2 \AA$ (Fig. S2). A similar result was reported by Chen et al. ${ }^{[18]}$ for 3D reconstruction of Au wedge sample.

Table 1: List of TEM image simulation parameters, phase change per atom, absolute defocus error and number of atoms deviation present on simulated nanoparticles with varying $t_{p} / t_{a c}$ 
ratios where carbon densities are $2.1 \mathrm{~g} / \mathrm{cc}$ and $3 \mathrm{~g} / \mathrm{cc}(*)$. Particle thickness $\left(\mathrm{t}_{\mathrm{p}}\right)=3.6 \mathrm{~nm}$ and amorphous carbon film thickness $\left(\mathrm{t}_{\mathrm{ac}}\right)=0.9,1.8,3.6,7.2$ and $14.5 \mathrm{~nm}$ respectively.

\begin{tabular}{|c|c|c|c|c|c|c|c|c|c|c|c|}
\hline \multirow[t]{2}{*}{$\begin{array}{l}\text { Sl. } \\
\text { No. }\end{array}$} & \multirow[t]{2}{*}{ Particle } & \multirow{2}{*}{$\begin{array}{l}\text { Lattice } \\
\text { param- } \\
\text { eter } \\
(\AA)\end{array}$} & \multirow{2}{*}{$\begin{array}{c}\text { Debye- } \\
\text { Waller factor } \\
\left(\AA^{2}\right)\end{array}$} & \multirow{2}{*}{$\begin{array}{c}\text { Phase } \\
\text { change } \\
\text { per } \\
\text { atom } \\
\text { (rad) }\end{array}$} & \multirow[t]{2}{*}{$\begin{array}{l}t_{\mathrm{p}} / \mathrm{t}_{\mathrm{ac}} \\
\text { ratios }\end{array}$} & \multirow{2}{*}{$\begin{array}{l}\text { Defocus } \\
\text { RMS error } \\
\text { (nm) }\end{array}$} & \multicolumn{5}{|c|}{$\begin{array}{c}\text { Number of atoms } \\
\text { deviation (\%) }\end{array}$} \\
\hline & & & & & & & \pm 1 & \pm 2 & \pm 3 & \pm 4 & \pm 5 \\
\hline \multirow[t]{6}{*}{1.} & \multirow[t]{6}{*}{$\mathrm{Ag}$} & \multirow[t]{6}{*}{4.079} & \multirow[t]{6}{*}{20} & \multirow[t]{6}{*}{0.13} & 4 & 0.14 & 7 & 0 & 0 & 0 & 0 \\
\hline & & & & & 2 & 0.20 & 14 & 0 & 0 & 0 & 0 \\
\hline & & & & & 1 & 0.24 & 21 & 1 & 0 & 0 & 0 \\
\hline & & & & & 0.5 & 0.26 & 42 & 2 & 0 & 0 & 0 \\
\hline & & & & & 0.25 & 0.52 & 55 & 17 & 5 & 0 & 0 \\
\hline & & & & & $0.25^{*}$ & 0.56 & 47 & 18 & 14 & 3 & 0 \\
\hline \multirow[t]{6}{*}{2.} & \multirow[t]{6}{*}{$\mathrm{Cu}$} & \multirow[t]{6}{*}{3.597} & \multirow[t]{6}{*}{5} & \multirow[t]{6}{*}{0.12} & 4 & 0.16 & 6 & 0 & 0 & 0 & 0 \\
\hline & & & & & 2 & 0.21 & 17 & 0 & 0 & 0 & 0 \\
\hline & & & & & 1 & 0.24 & 34 & 2 & 0 & 0 & 0 \\
\hline & & & & & 0.5 & 0.31 & 41 & 2 & 0 & 0 & 0 \\
\hline & & & & & 0.25 & 0.35 & 48 & 17 & 2 & 0 & 0 \\
\hline & & & & & $0.25^{*}$ & 0.46 & 48 & 17 & 6 & 4 & 2 \\
\hline \multirow[t]{6}{*}{3.} & \multirow[t]{6}{*}{$\mathrm{Au}$} & \multirow[t]{6}{*}{4.065} & \multirow[t]{6}{*}{20} & \multirow[t]{6}{*}{0.19} & 4 & 0.10 & 9 & 0 & 0 & 0 & 0 \\
\hline & & & & & 2 & 0.15 & 14 & 0 & 0 & 0 & 0 \\
\hline & & & & & 1 & 0.18 & 31 & 0 & 0 & 0 & 0 \\
\hline & & & & & 0.5 & 0.19 & 36 & 1 & 0 & 0 & 0 \\
\hline & & & & & 0.25 & 0.27 & 41 & 3 & 0 & 0 & 0 \\
\hline & & & & & $0.25 *$ & 0.29 & 34 & 17 & 2 & 0 & 0 \\
\hline
\end{tabular}

On the other hand, comparing the focus map (column 4 of Fig. 1) and histograms of the normalised defocus values (column 1 of Fig. S2) of supported and unsupported parts of the Ag disc reveal that with increasing carbon thickness and density, the variation in focus values of the atomic columns increases. Therefore, it can be stated that the presence of amorphous carbon support modifies the exit surface geometry of a nanoparticle.

The phase shift per atom is influenced by the electron dose rate and thermal vibration of the atomic column $^{[30]}$. This value was calculated by simulating a wedge sample structure with varying number of atoms from one to four with different Debye-Waller factors. Column 5 of Fig. 1 illustrates the mass thickness map, which represents the number of atoms in each atomic column. The plot of phase of FCW with respect to the number of atom for all tp/tac ratio is shown in column 4 of Fig. S1. Mass thickness map of the unsupported region indicates two types of atomic columns, those are periodically arranged and consist of 9 and 10 atoms respectively, that is evident in the histogram shown in row 1, column 2 of Fig. S2. The carbon-supported regions exhibit the randomness in atom counts. The error in atom counts for each column increases with increasing supporting carbon thickness and density which is evident in the histograms of column 2 of Fig. S2. Therefore it can be stated that the amorphous carbon support randomly modifies the atom count in atomic columns within the imaged region. 
Chen et al. reported that the valley/background wave between atomic columns plays a significant role in normalizing reconstructed exit waves to calculate exact focus by MPI criterion and FCW at the exit

surface $^{[18]}$. The selection of valley waves is very crucial in the tomographic reconstruction of nanoparticle supported on amorphous carbon film and the procedure used in the present study is described in the methods section. Two different options, such as single and average valley waves surrounding the projected atomic columns, were tested. To compare the effectiveness between the options of valley wave selections, the imaged region marked with green rectangle box in row 3, column 2 of Fig. 1, was nominated. Changes in phase value of background (valley wave) and atomic columns (FCW) after normalization and propagation are plotted in Fig 2 A and B, respectively. In Fig. 2A, the blue (single valley wave) and the red line (average valley wave) are merged in the unsupported region because the crystal is homogeneous; hence both valley waves experience the same mean inner potential ${ }^{[18]}$. In the carbonsupported region, the deviation associated with the blue line is more as compared to the red line. Since the four valley waves experience different mean inner potentials, their average value reduces the variation; hence the error in phase value of the average valley wave is less as compared to single valley wave. In Fig. 2B, the zig-zag pattern shown in the phase of FCWs in the unsupported region is due to the alternating periodic array of 9 and 10 atoms in atomic columns. Whereas in carbon-supported region, the zig-zag pattern of both blue and red lines is modified both in amplitude and periodicity. Here also blue line deviates more than the red line. Therefore, it can be stated that the error in atom count deviates from actual value in case of selecting single valley waves than the average one.

The error in focus map calculation occurs due to the deviation in both the phases of valley wave and FCW of atomic columns, as shown in Fig. $2 \mathrm{C}$ and $\mathrm{D}$. The phase of valley wave depends upon the combination of phase shift due to mean inner potential of nanoparticle and amorphous carbon support $^{[31]}$. The expression for the phase of valley wave $\left(\Phi_{\text {valley }}\right)$ is given as,

$\Phi_{\text {valley }}=\Phi_{\text {nanoparticle }}+\Phi_{\text {carbon }}$

$\Phi_{\text {valley }}=\sigma\left(V_{p} t_{p}+V_{a c} t_{a c}\right)$

Where,

$\sigma=$ relativistic electron interaction constant

$V_{p}=$ mean inner potential of the nanoparticle

$t_{p}=$ thickness of nanoparticle along electron bean direction

$\mathrm{V}_{\mathrm{ac}}=$ mean inner potential of the amorphous carbon support

$t_{\mathrm{ac}}=$ thickness of amorphous carbon support 
The phase of valley wave is calculated for the nanostructure shown in row 3, column 1 of Fig. 1 using the above equation to validate the results presented in Fig. 2A. The $\sigma$ for $300 \mathrm{keV}$ accelerating voltage is $0.00065 \mathrm{rad} /(\mathrm{eV} \AA)^{[18]}, \mathrm{V}_{\mathrm{p}}$ for Ag nanoparticle is $23.2 \mathrm{~V}^{[32]}$, as per simulation $\mathrm{t}_{\mathrm{ac}}$ and $\mathrm{t}_{\mathrm{p}}$ both are $36 \AA$ and $\mathrm{V}_{\mathrm{ac}}$ for amorphous carbon $(2.1 \mathrm{~g} / \mathrm{cc})$ is $10.7 \mathrm{~V}^{[31]}$. The modulus of the phase of the valley wave due to an Ag nanoparticle is $0.542 \mathrm{rad}$ calculated using the above equation, which is very close to the phase value of valley wave $(0.5 \mathrm{rad})$ obtained from the analysis of the unsupported region of nanoparticle as shown in Fig. 2A. The modulus of single valley wave of supported region of nanoparticle shown in Fig. 2A varies from $0.03-0.27 \mathrm{rad}$. The calculated modulus of phase of valley wave due to amorphous carbon is 0.25 rad, which lies within the above-mentioned range. Therefore, it is confirmed that the amorphous carbon presence modifies the phase of the valley wave. The error in phase of valley wave affects the calculation of exact focus for an atomic column, which in turn modifies the phase of FCWs and number of atoms (atom count) in atomic columns. This error can be minimized by local averaging of valley waves around the atomic columns.

For quantitative analysis, focus and mass thickness calculation errors of simulated Ag nanoparticle supported on amorphous carbon having $t_{p} / t_{a c}=1$ were estimated for single and average valley wave selections and presented in Fig. $2 \mathrm{C}$ and D, respectively. The root mean square (RMS) error in carbonsupported region of the focus map was calculated by taking an unsupported region of $t_{p} / t_{a c}=4$ as a reference, which has a periodic array of normalized defocus values of $-1 \AA$ and $+1 \AA$ as shown in the histogram of Fig. S2A. This unsupported region of $t_{p} / t_{a c}=4$ was selected to nullify the error that occurs at the supported/unsupported interface with increasing carbon thickness. The error in atom count was also estimated by considering the same unsupported region as reference. The unsupported region has a periodic array of 9 and 10 atoms in the atomic column, as shown in histogram of row 1, column 2 of Fig. S2. The deviation in atom counts in each atomic column w.r.t the atom count of corresponding atomic columns in the unsupported region as mentioned above, was considered as mass thickness calculation error. The percentage of atomic columns with error in atom count was calculated and presented in Fig. 2D. Considering Fig. $2 \mathrm{C}$ and $\mathrm{D}$, it can be stated that selecting average valley wave over the single one minimizes the errors in focus and mass thickness calculation. Averaging valley waves reduces the deviation of phase error in the valley wave of the supported region w.r.t the unsupported region; hence, the error in finding exact focus and FCW reduces, leading to a reduction of errors in atom count.

Other than $\mathrm{Ag}(Z=47)$, the influence of amorphous carbon support is further investigated for simulated disc-shaped nanoparticles with different atomic numbers such as $\mathrm{Cu}(Z=29)$ and $\mathrm{Au}(Z=79)$. For both $\mathrm{Cu}$ and Au nanoparticles, the average valley wave was considered for calculation of MPI. Similarly, results related to $\mathrm{Cu}$ (Fig. S4, S5) and Au (Fig. S6, S7) nanoparticles are presented in the supplementary section. The phase change per atom for $\mathrm{Ag}, \mathrm{Cu}$ and Au subjected to different electron doses (related to DebyeWaller factor) are calculated using mass circles in Argand plane (Fig. S8) and tabulated in Table 1.

Fig. 3 represents focus $(A, C$, and $E)$ and mass thickness (B, D, and $F$ ) error calculations for different $t_{p} / t_{a c}$ ratios and different carbon densities of simulated $\mathrm{Ag}, \mathrm{Cu}$, and $\mathrm{Au}$ nanoparticles. The images of $\mathrm{Ag}$ and $\mathrm{Au}$ 
nanoparticles were simulated with Debye-Waller factor of $20 \AA^{2}\left(\sim 10^{4} \mathrm{e} / \mathrm{nm}^{2}\right.$ sec electron dose rate $\left.{ }^{[30]}\right)$ and Cu with Debye-Waller factor of $5 \AA^{2}\left(\sim 5^{\star} 10^{2} \mathrm{e} / \mathrm{nm}^{2} \mathrm{sec}{ }^{[30]}\right)$ and listed in Table 1. D. Van Dyck et al. ${ }^{[30]}$ reported that phase shift per atom for an element decreases with an increase in electron dose rate during image acquisition and increases with an increase in atomic number. Increasing the electron dose rate increases the thermal vibration of atoms, which in turn flatten the projected mean inner potential experienced by the incoming electron during TEM imaging. Selecting a higher Debye-Waller factor means considering higher thermal vibration of atoms therefore, results in a decrease of phase shift per atom. Since $\mathrm{Ag}$ has a higher atomic number than $\mathrm{Cu}$, so, different electron dose rate for $\mathrm{Cu}$ was selected. This selection results in almost similar error in mass thickness calculation for both $\mathrm{Ag}$ and $\mathrm{Cu}$. In case of $\mathrm{Ag}$ and Au same electron dose rate was selected, so the mass thickness calculation error was less in case of Au due to a higher phase shift per atom than Ag. From the overall results presented in Fig. 3 A-F, it can be stated that irrespective of the atomic number of a constituent element of nanoparticle, the focus and mass thickness calculation error increases with increasing supporting carbon thickness and/or density.

The schematic shown in Fig. 3G is used to exhibit the effect of amorphous carbon support on the mass circle, exact focus, and phase of $\theta^{\prime}$ in the Argand plane regarding a disc-shaped nanoparticle containing the same atom count in all the atomic columns and half of which is supported on amorphous carbon. The red dot at $(1,0)$ represents the vacuum wave. The green dot in mass circle shows the FCW for singleatom having phase angle $\theta$. The atom counts in atomic columns can be obtained by dividing the phase of $\theta^{\prime}$ with phase shift per atom of $\theta$. The blue dots in the mass circle represent the FCW of atomic columns having the same number of atoms and the phase of $\theta^{\prime}$. The blue dots are concentrated on the mass circle indicates that the atomic columns are in the same focus or having the same flat exit surface in the unsupported region. The yellow dots are the defocused wave due to amorphous carbon support. The amorphous carbon induced defocused waves is away from blue dots in mass circle, which indicates that the waves are defocused from the exact focus of FCWs. The yellow dots at the right side of FCWs (blue dots) have smaller phase angle $\left(\theta^{\prime}-n \theta\right)$ on contrary to the yellow dots at the left side have larger phase angle $\left(\theta^{\prime}+n \theta\right)$. The larger phase angle corresponds to an increased error in atom count $(n)$ in atomic columns and vice versa. The number of atoms increased or decreased in an atomic column depends upon phase shift per atom. It can be stated that the scattering of FCWs on mass circle indicates the amorphous carbon support affects surface roughness as well as atom count.

Considering simulated results together, it can be summarized that the presence of amorphous carbon support modifies the nanoparticle's exit surface roughness due to the focus and mass thickness calculation errors. Consequently, tomographic reconstruction by combining information of focus map and mass thickness map for carbon-supported nanoparticle becomes distorted as compared to unsupported one.

\section{Experimental results}

After analysing the results related to tomographic reconstruction of simulated nanoparticles supported on amorphous carbon, the same procedure was applied to experimental Ag nanoparticle supported on 
amorphous carbon film. A silver nanoparticle of around $15 \mathrm{~nm}$ diameter at lattice resolution viewed along $<110>$ is presented in Fig. 4A from a set of focal series images. Since this nanoparticle was partly supported on carbon film, so, it allowed selecting different regions with varying $t_{p} / t_{a c}$ ratios. The rectangular yellow-colored region in right upper portion of Fig. 4A seems to be completely supported on carbon film. The amplitude and phase of reconstructed complex-valued exit waves are presented in Fig. $4 \mathrm{~B}$ and $4 \mathrm{C}$, respectively. The rectangular yellow coloured region in the left lower portion of Fig. 4A seems to be partly supported. The amplitude and phase of the reconstructed complex-valued exit wave for this portion are shown in Fig. 4D and 4E, respectively. Within the lower rectangular region, the edge portion marked as region 1 seems to be completely unsupported. Region 2 seems to have thinner carbon support (higher $t_{p} / t_{a c}$ ) than region 3 . These three regions were nominated for atomically resolved tomographic reconstruction.

Fig. 5 represents the results related to tomographic reconstruction of region 1,2 and 3 of Ag nanoparticle. Since experimental Ag nanoparticle was oriented in $\langle 110\rangle$, for valley wave selection, averaging of six valley waves surrounding each atomic column in the image plane was done. Fig. 5(A-E) represents the plot of the mass circle and phase $\theta^{\prime}$ in Argand plane, focus map, mass thickness map, the histogram of focus values from focus map, and atom counts from mass thickness map respectively for region 1. Similarly, Fig. 5(F-J) and (K-O) represent the results related to regions 2 and 3, respectively. The phase shift per atom for experimental Ag nanoparticle was calculated as 0.09 rad (also reported in Fig. 4 of ${ }^{[30]}$ ) using a wedge sample with 4 atoms along [110] direction using Debye-Waller factor of $36 \AA^{2}$ and is shown in mass circle of Fig. S8 D. The thickness (focus) accuracy (determined according to ${ }^{[9]}$ ) is obtained by finding the average standard deviation of a discrete set of peaks in the histogram of focus map (Fig. $5 \mathrm{D}, \mathrm{I}, \mathrm{N}$ ). The values are $0.4 \AA, 0.6 \AA$, and $1 \AA$ for regions 1,2 , and 3 , respectively as mentioned in Table 2. The thickness accuracies for all three regions were less than the interatomic spacing of $2.9 \AA$ for Ag crystal along $\langle 110\rangle$ direction which reveals that the thickness direction is atomically resolved. Since maximum number of atomic columns within mass thickness map of region 1 (Fig. 5 C, E) has 8 atoms, therefore corresponding average thickness of region 1 along viewing direction was calculated as $2.3 \mathrm{~nm}$. Similarly, for region 2 (Fig. $5 \mathrm{H}, \mathrm{J}$ ) and 3 (Fig. 5M, O) maximum number of atomic columns contains 13 and 12 atoms, so, corresponding thicknesses along viewing direction were calculated as 3.77 $\mathrm{nm}$ and $3.48 \mathrm{~nm}$, respectively.

Table 2: List of electron dose, phase change per atom, phase and defocus error of experimental Ag nanoparticle 


\begin{tabular}{|c|c|c|c|c|c|c|}
\hline $\begin{array}{c}\text { Sl. } \\
\text { No. }\end{array}$ & Region & $\begin{array}{c}\text { Electron } \\
\text { dose } \\
\text { rate } \\
\left(\mathrm{e} / \mathrm{nm}^{2} \text { sec }\right)\end{array}$ & $\begin{array}{c}\text { Debye- } \\
\text { Waller } \\
\text { factor } \\
\left(\AA^{2}\right)\end{array}$ & $\begin{array}{c}\text { Phase change per } \\
\text { atom (rad) }\end{array}$ & $\begin{array}{c}\text { Phase } \\
\text { accuracy } \\
(\text { SD) (rad) }\end{array}$ & $\begin{array}{l}\text { Thickness } \\
\text { accuracy } \\
(S D)(\AA)\end{array}$ \\
\hline 1. & $\begin{array}{c}\text { Region } \\
1\end{array}$ & $\begin{array}{c}\text { Approx. } \\
8 * 10^{5}\end{array}$ & 36 & 0.09 & 0.029 & 0.4 \\
\hline 2. & $\begin{array}{c}\text { Region } \\
2\end{array}$ & $\begin{array}{c}\text { Approx. } \\
8 * 10^{5}\end{array}$ & 36 & 0.09 & 0.023 & 0.6 \\
\hline 3. & $\begin{array}{c}\text { Region } \\
3\end{array}$ & $\begin{array}{c}\text { Approx. } \\
8 * 10^{5}\end{array}$ & 36 & 0.09 & 0.02 & 1.0 \\
\hline
\end{tabular}

The plots of intensity profile as a function of defocus for three regions are presented in Fig. S9 (A-C). The phase accuracy (determined according to ${ }^{[9]}$ ) was obtained by finding the average standard deviation related to phase of FCWs for each number of atoms shown in Fig. S9 D-F. The phase accuracy for regions 1,2 , and 3 are $0.029 \mathrm{rad}, 0.023 \mathrm{rad}$, and 0.02 rad, respectively.

Mass circles in Argand plane (Fig, $5 \mathrm{~A}$, F, and $\mathrm{K}$ ) provide direct intimation regarding the presence of amorphous carbon as described for simulated nanoparticles. Here red dots represent FCWs, and points $(1,0)$ represent vacuum waves related to respective regions. The FCWs lie on the mass circle of region 1 confirms that the portion is unsupported. FCWs are scattered to some extent from mass circle in region 2 and largely scattered in region 3 which confirm that the supporting carbon film thickness is less for region 2 than region 3.

The atomically resolved tomograms of regions 1,2 and 3 of the experimental nanoparticle are presented in Fig. $6 \mathrm{~A}-\mathrm{C}$, respectively. The average thickness of the holey carbon grid used for the support in this experiment is around 3-4 nm. Since region 3 was fully supported on carbon film so, the $t_{p} / t_{a c}$ ratio here was considered approximately 1 . The viewing directions for both simulated and experimental $\mathrm{Ag}$ nanoparticles are different. However, the tomograms of region 3 and the simulated nanostructure with $t_{p} / t_{a c}=1$ can still be qualitatively compared. Fig. $6 \mathrm{D}$ represents the tomogram of the simulated nanostructure $\left(t_{p} / t_{a c}=1\right)$ shown in row 3 , column 1 of Fig. 1 . Here the rectangular region of the unsupported part of the disc-shaped nanoparticle resembles accurate tomographic reconstruction, but the supported part depicts artificial roughness induced due to the presence of carbon support. A similar kind of roughness is also visible in the tomogram of region 3 presented in Fig. $6 \mathrm{C}$, which establishes the distortion in tomographic reconstruction of nanoparticle in the presence of the supporting amorphous carbon film.

So far, the influence of amorphous carbon support on the tomographic reconstruction of simulated and experimental nanoparticles was discussed. Now, the challenges of tomographic reconstruction of nanoparticles with amorphous carbon support need to be enlightened. In simulations, one half of the disc-shaped nanoparticle was made carbon-supported, and another was unsupported. The disc-shaped particle was considered for the flatness of its exit surface. By selecting an unsupported region as a reference, the focus and mass thickness calculation errors in the supported region was calculated for the 
simulated cases, but in the experimental case, it cannot be done because shape of the nanoparticle was unknown prior to the reconstruction. Moreover, error due to carbon support distorts the periodicity in viewing direction, so, to reconstruct tomogram of a nanoparticle supported on carbon film, fine tuning of focus needs to be done in a way that the focus values be the multiple of interatomic spacing.

The influence discussed until now may also be observable in case of cross-sectional samples like thin films where amorphous glue is used for TEM sample preparation. The leftover of amorphous glue on top and/or bottom surface may produce artefacts in a similar way as amorphous carbon support because it was already reported that this leftover glue produces stain artefacts within images of thin films like carbon support produces in images of nanoparticles ${ }^{[33]}$.

\section{Conclusions}

In this present work, the influence of amorphous carbon support on the atomically resolved tomographic reconstruction of simulated and experimental nanoparticles from a single projection was examined. It was established that to select the valley wave during normalization of reconstructed exit wave, averaging surrounding the atomic column on the image plane minimizes errors. With increasing carbon thickness and/or density, focus and mass thickness calculation errors related to the tomographic reconstruction of nanoparticle increase, which in turn induce artificial surface roughness and modify atom count in atomic columns. However, the atomic number of a constituent element of nanoparticle does not have any significant influence on supporting carbon film-induced errors.

\section{Methods}

\section{Tomographic reconstruction of simulated disc shape nanoparticles on an amorphous carbon support}

\subsection{Structure of simulated nanoparticles}

To mimic experimental procedure, disc-shaped nanoparticles ( $4.5 \mathrm{~nm}$ diameter and $3.6 \mathrm{~nm}$ thickness) of three different elements $\mathrm{Ag}, \mathrm{Cu}$ and $\mathrm{Au}$ of same crystal structure (FCC) were constructed along [001] direction by VESTA ${ }^{T M}{ }^{[34]}$ software. The disk shape was chosen to avoid thickness variation across the simulated nanoparticle. For all the elements, one half of the simulated nanoparticle was placed on amorphous carbon supporting film while the other half was kept hanging, as shown in the first column of Fig. 1. Similarly, like previous literature ${ }^{[35]}$, a fixed $2 \AA$ gap was always maintained between the nanoparticle and the supporting film. Supporting amorphous carbon (ac) film was simulated using molecular dynamics by the liquid quenching method as described previously ${ }^{[27]}$. Carbon atoms corresponding to a density of $2.1 \mathrm{~g} / \mathrm{cc}$ and $3 \mathrm{~g} / \mathrm{cc}$ were randomly modeled in a block of $10 \mathrm{~nm} \times 10 \mathrm{~nm} \times$ $30 \mathrm{~nm}$. The simulated nanoparticles of $\mathrm{Ag}, \mathrm{Cu}$, and Au with a thickness of $3.6 \mathrm{~nm}$ were placed on amorphous carbon film (density $=2.1 \mathrm{~g} / \mathrm{cc}$ ) with varying thicknesses of $0.9,1.8,3.6,7.2$ and $14.5 \mathrm{~nm}$ to demonstrate the effect of ac supporting film thickness on the atomically resolved tomographic reconstruction of the nanoparticle. 
Moreover, to study the effect of amorphous carbon density on the tomographic reconstruction, the nanoparticles were similarly placed on $14.5 \mathrm{~nm}$ amorphous carbon film with a higher density $(3 \mathrm{~g} / \mathrm{cc})$. Whereas Ag and Au disc shape particles have an alternating periodic array of 9 and 10 atoms, Cu has 10 and 11 atoms respectively. The bottom surface of the disc-shaped nanoparticles of the present study has ABAB stacking. The thickness difference between $A$ and $B$ layers at the bottom surface is approx. $2 \AA$. We demonstrate different structures with varying $t_{p} / t_{a c}$ ratio, where $t_{p}$ and $t_{a c}$ are nanoparticle and supporting amorphous carbon film thickness, respectively. All the simulated structures are presented in the first column of Fig. 1 for Ag nanoparticle, Fig. S4 for Cu nanoparticle and Fig. S6 for Au nanoparticle.

\subsection{TEM image simulation}

The QSTEM ${ }^{[36]}$ software was used to simulate high-resolution lattice images of all the simulated nanostructures with different $t_{p} / t_{a c}$ ratios along $\langle 100\rangle$ zone axis, as described previously ${ }^{[35]}$. The highresolution lattice images were simulated using the multislice algorithm. TEM experimental parameters used in the simulation are as follows: accelerating voltage $=300 \mathrm{kV}$, spherical aberration coefficient (Cs) $=-0.003 \mathrm{~mm}$, Scherzer defocus $=9.3 \mathrm{~nm}$, energy spread $=0.6 \mathrm{eV}$, objective aperture radius $=38 \mathrm{mrad}$. An overall sampling of $0.0834 \AA$ per pixel and slice thickness of $0.1 \AA$ was used for all the cases to obtain better lateral and depth resolution. The Debye-Waller factor was applied to all the simulated images to incorporate the effect of the electron dose rate.

\subsection{In-line holography of simulated nanoparticles}

A set of focal series comprises 21 images was simulated in the defocus range from $-0.7 \mathrm{~nm}$ to $19.3 \mathrm{~nm}$ with a defocus step of $1 \mathrm{~nm}$. The other parameters are chosen like defocus spread $=3.6 \mathrm{~nm}$ and convergence angle $=0.1 \mathrm{mrad}$. These focal series images were used to reconstruct the complex-valued exit face wave by FRWR plugin ${ }^{[22]}$ of Digital Micrograph ${ }^{T M}$ for all the simulated nanostructures.

\subsection{Tomographic reconstruction of simulated nanoparticles:}

The exit wave reconstructed by in-line holography contains the field information such as amplitude and phase, which is applicable for tomographic reconstruction using channelling theory. In line 3D holography method reported by Chen et al. ${ }^{[9]}$ was used in this present work for tomographic reconstruction from a single projection. For the presence of supporting amorphous film with the nanoparticle, the modification was mainly incorporated while selecting valley wave. The necessary steps for tomographic reconstruction from single projection ${ }^{[9]}$ are as follows:

a. Selection of Valley wave or background wave

b. Determination of exact focus map or bottom surface geometry using MPI criteria

c. Plotting mass circle and phase of $\theta^{\prime}(\mathrm{rad})$

d. Calculation of phase change $\theta(\mathrm{rad})$ per atom

e. Plotting mass thickness map 


\section{f. Plotting tomograms}

Modification in the selection of Valley wave or background wave:

The background wave selection is the significant parameter that affects the z-height and number of atoms in the respective column. For valley wave selection, there are four valley waves present in the phase between the atomic columns where the Ag crystal structure viewed along [001]. To select the valley wave appropriately, two different options were tested: 1) Single valley wave out of four valley waves surround the atomic column 2) Average of four valley waves surround the atomic column. Nanostructure containing Ag nanoparticle with $\mathrm{t}_{\mathrm{p}} / \mathrm{t}_{\mathrm{ac}}=1$ and amorphous carbon density of $2.1 \mathrm{~g} / \mathrm{cc}$ was chosen to study the effect of valley wave selection on tomographic reconstruction. The result related to the single valley wave is presented in Fig. S3 and average valley wave in row 3, column 3-5 of Fig. 1.

\section{Tomographic reconstruction of experimental Ag nanoparticle supported on holey carbon}

\subsection{High-resolution lattice imaging and in-line holography:}

To perform the TEM experiment, Ag nanoparticles (YF26042016, PlasmaChem Gmbh, $0.1 \mathrm{mg} / \mathrm{mL}$ aqueous suspension, $10 \mathrm{~nm}$ ) were dispersed in an ethanol solution, followed by ultra-sonication for $1 \mathrm{hr}$ to avoid agglomeration. Finally, the solution was drop cast on a lacey carbon supporting film deposited on TEM Cu grid (Agar Scientific., AGS166).

High-resolution lattice images were acquired using FEI TITAN ${ }^{3}$ Themis microscope operated at $300 \mathrm{keV}$, equipped with a field emission gun (FEG) and a $2 \mathrm{~K} \times 2 \mathrm{~K}$ CCD camera (FEl, BM-Ceta). The images of Ag nanoparticle were acquired along $<110>$ crystallographic orientation with parallel illumination and $C_{s}$ value of about $-0.003 \mathrm{~mm}$. The electron dose rate was maintained at approx. $8 * 10^{5} \mathrm{e} / \mathrm{nm}^{2} \mathrm{sec}$. A set of 35 images were recorded at different defocuses ranging from - 17 to $18 \mathrm{~nm}$ with a step size of $1 \mathrm{~nm}$. Two regions of Ag nanoparticle were taken for in-line holography, as shown in Fig. 4A marked with yellow rectangles. A stack of 21 focal series images was used for each region. Similar to simulation, FRWR software tool used for exit wave reconstruction during experimental analysis also.

\subsection{Tomographic reconstruction of experimental nanoparticle:}

Tomographic reconstruction of experimental nanoparticle was done in a similar fashion as of the simulated ones.

\section{Declarations}

\section{Acknowledgements:}

PB and SB are very much thankful to Prof. Fu Rong Chen and Dr. Shih-Wei Hung of City University Hong Kong for their help in understanding In-line 3D holography. The financial assistance from the Science and Engineering Research Board (SERB), core research grant project no. EMR/F/2017/001510 is highly 
acknowledged. AJS would like to thank the IMEYMAT Institute and the Spanish Ministry of Education and Culture for the concessions of Grants ICARO-173873 and FPU16-04386.

\section{Author contributions:}

SB conceptualized the problem. TEM experiment was done by the University of Cádiz group. Simulation and data analysis were done by the IIT Madras group.

\section{Competing interest:}

The authors declare no competing financial interest.

\section{References}

[1] R. Taylor, S. Coulombe, T. Otanicar, P. Phelan, A. Gunawan, W. Lv, G. Rosengarten, R. Prasher, H. Tyagi, J. Appl. Phys. 2013, 113, 011301.

[2] P. R. Bueno, C. Gabrielli, Nanostructured Materials for Electrochemical Energy Production and Storage, Springer US, Boston, MA, 2009.

[3] M. De, P. S. Ghosh, V. M. Rotello, Adv. Mater. 2008, 20, 4225.

[4] A. D. Servin, J. C. White, Nanolmpact 2016, DOI 10.1016/j.impact.2015.12.002.

[5] R. Ghosh Chaudhuri, S. Paria, Chem. Rev. 2012, 112, 2373.

[6] I. Khan, K. Saeed, I. Khan, Arab. J. Chem. 2019, DOI 10.1016/j.arabjc.2017.05.011.

[7] R. Feynman, Eng. Sci. 22-361959.

[8] S.-H. Kim, P. W. Kang, O. O. Park, J.-B. Seol, J.-P. Ahn, J. Y. Lee, P.-P. Choi, Ultramicroscopy 2018, 190, 30 .

[9] F. R. Chen, D. Van Dyck, C. Kisielowski, Nat. Commun. 2016, 7, 462.

[10] S. Vilayurganapathy, A. Devaraj, R. Colby, A. Pandey, T. Varga, V. Shutthanandan, S. Manandhar, P. Z. El-Khoury, A. Kayani, W. P. Hess, S. Thevuthasan, Nanotechnology 2013, 24, 095707.

[11] S. V. N. T. Kuchibhatla, V. Shutthanandan, T. J. Prosa, P. Adusumilli, B. Arey, A. Buxbaum, Y. C. Wang, T. Tessner, R. Ulfig, C. M. Wang, S. Thevuthasan, Nanotechnology 2012, 23, 215704.

[12] D. Van Dyck, F.-R. Chen, Nature 2012, 486, 243.

[13] B. D. A. Levin, E. Padgett, C. C. Chen, M. C. Scott, R. Xu, W. Theis, Y. Jiang, Y. Yang, C. Ophus, H. Zhang, D. H. Ha, D. Wang, Y. Yu, H. D. Abruña, R. D. Robinson, P. Ercius, L. F. Kourkoutis, J. Miao, D. A. Muller, R. Hovden, Sci. Data 2016, DOI 10.1038/sdata.2016.41. 
[14] M. C. Scott, C. C. Chen, M. Mecklenburg, C. Zhu, R. Xu, P. Ercius, U. Dahmen, B. C. Regan, J. Miao, Nature 2012, 483, 444.

[15] E. Padgett, R. Hovden, J. C. Dasilva, B. D. A. Levin, J. L. Grazul, T. Hanrath, D. A. Muller, Microsc. Microanal. 2017, DOI 10.1017/S1431927617012764.

[16] R. F. Egerton, P. Li, M. Malac, Micron 2004, 35, 399.

[17] M. C. Scott, C.-C. Chen, M. Mecklenburg, C. Zhu, R. Xu, P. Ercius, U. Dahmen, B. C. Regan, J. Miao, Nature 2012, 483, 444.

[18] F.-R. Chen, C. Kisielowski, D. Van Dyck, Micron 2015, 68, 59.

[19] L. J. Allen, W. McBride, N. L. O'Leary, M. P. Oxley, Ultramicroscopy 2004, 100, 91.

[20] S. Bhattacharyya, C. T. Koch, M. Rühle, Ultramicroscopy 2006, 106, 525.

[21] M. Op De Beeck, D. Van Dyck, Ultramicroscopy 1996, 64, 153.

[22] C. T. Koch, Ultramicroscopy 2008, 108, 141.

[23] D. Van Dyck, M. Op De Beeck, Ultramicroscopy 1996, 64, 99.

[24] A. Wang, F. R. Chen, S. Van Aert, D. Van Dyck, Ultramicroscopy 2010, 110, 527.

[25] A. Wang, F. R. Chen, S. Van Aert, D. Van Dyck, Ultramicroscopy 2012, 116, 77.

[26] F. R. Chen, C. Kisielowski, D. Van Dyck, Adv. Struct. Chem. Imaging 2017, 3, 8.

[27] M. Settem, P. Rajak, M. Islam, S. Bhattacharyya, Nanoscale 2017, 9, 17054.

[28] F.-R. Chen, D. Van Dyck, C. Kisielowski, H. Calderon, Microsc. Microanal. 2019, 25, 462.

[29] F.-R. Chen, D. Van Dyck, C. Kisielowski, S. Helveg, H. Calderon, A. Kirkland, in Eur. Microsc. Congr. 2016 Proc., Wiley-VCH Verlag GmbH \& Co. KGaA, Weinheim, Germany, 2016, pp. 25-26.

[30] D. Van Dyck, I. Lobato, F. R. Chen, C. Kisielowski, Micron 2015, 68, 158.

[31] M. Wanner, D. Bach, D. Gerthsen, R. Werner, B. Tesche, Ultramicroscopy 2006, 106, 341.

[32] A. Sanchez, M. A. Ochando, J. Phys. C Solid State Phys. 1985, DOI 10.1088/0022-3719/18/1/011.

[33] M. Islam, P. Rajak, S. K. Yadav, S. Chanda Roy, S. Bhattacharyya, J. Alloys Compd. 2020, 813, 152232.

[34] K. Momma, F. Izumi, J. Appl. Crystallogr. 2011, DOI 10.1107/S0021889811038970. 
[35] P. Rajak, C. T. Koch, S. Bhattacharyya, Ultramicroscopy 2019, DOI 10.1016/j.ultramic.2019.02.012.

[36] C. Koch, Dissertation 2002.

\section{Figures}

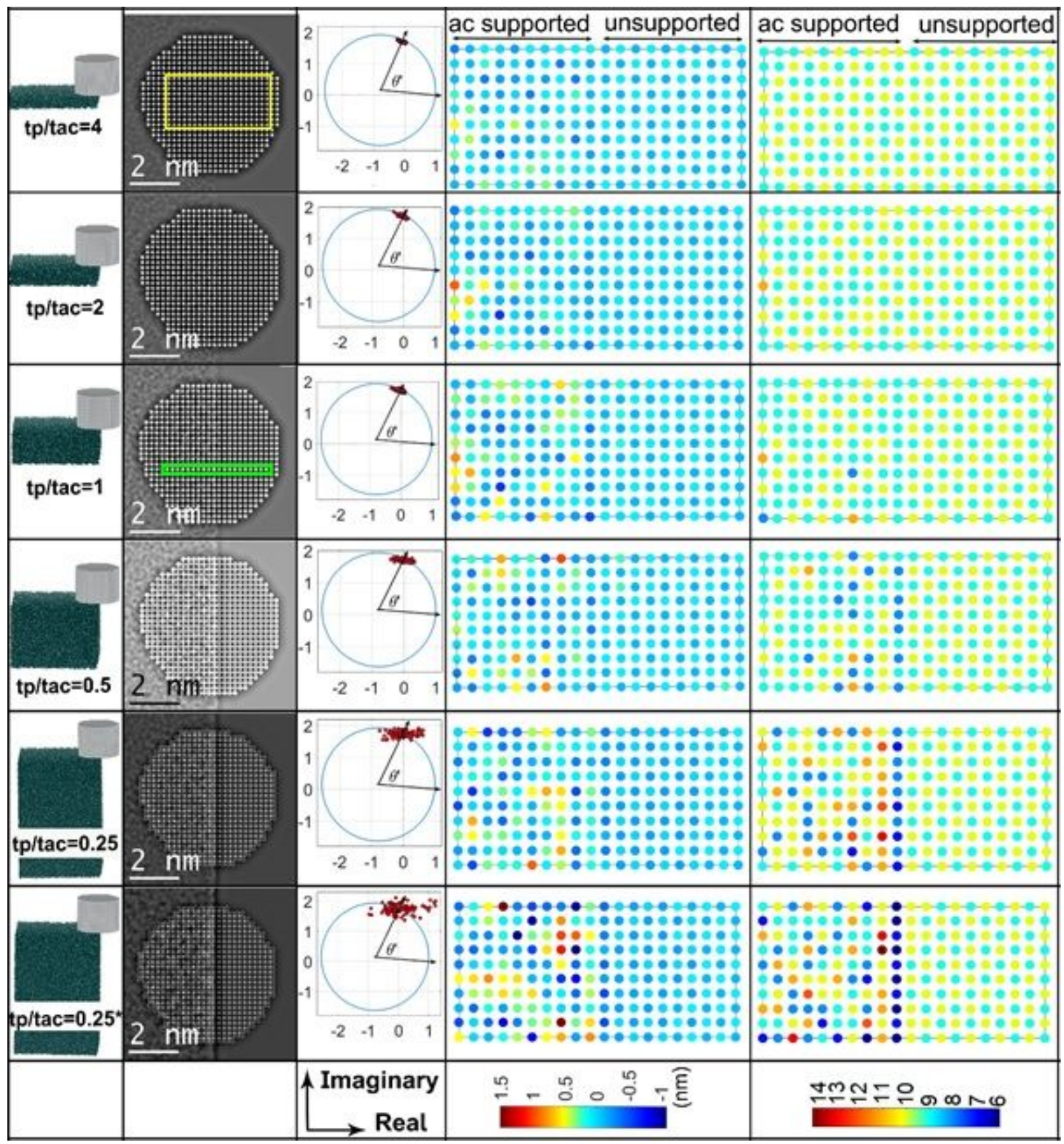

Figure 1

Effect of amorphous carbon support on 3D reconstruction of Ag particle. Each row represents the results related to the structure shown in column 1 of that row. Amorphous carbon density of the supporting film was taken as $2.1 \mathrm{~g} / \mathrm{cc}$ for row 1-5 and $3 \mathrm{~g} / \mathrm{cc}$ for row 6 as marked by "*”. Column 1: simulated structures of Ag nanoparticle supported on varying carbon thickness and density ( $2.1 \mathrm{~g} / \mathrm{cc}$ and $3 \mathrm{~g} / \mathrm{cc})$ along [001] 
direction. Column 2: Reconstructed phases of the complex valued exit wave function. Column 3: Plot of the mass circle and phase of $\theta^{\prime}$ of the simulated structures in column 1. Red dots are focus corrected waves (FCWs) and wave at $(1,0)$ in this plot is the vacuum wave. $X$ and $Y$ axis represent real and imaginary part and indicated in last row of column 3. Column 4: Normalised focus map of the imaged regions of simulated structures as marked by the yellow rectangle in (row 1, column 2). Respective colour bar is shown in (row 7, column 4). Column 5: Mass thickness map showing number of atoms in each atomic column of simulated Ag structures. Respective colour bar is shown in (row 7, column 5).
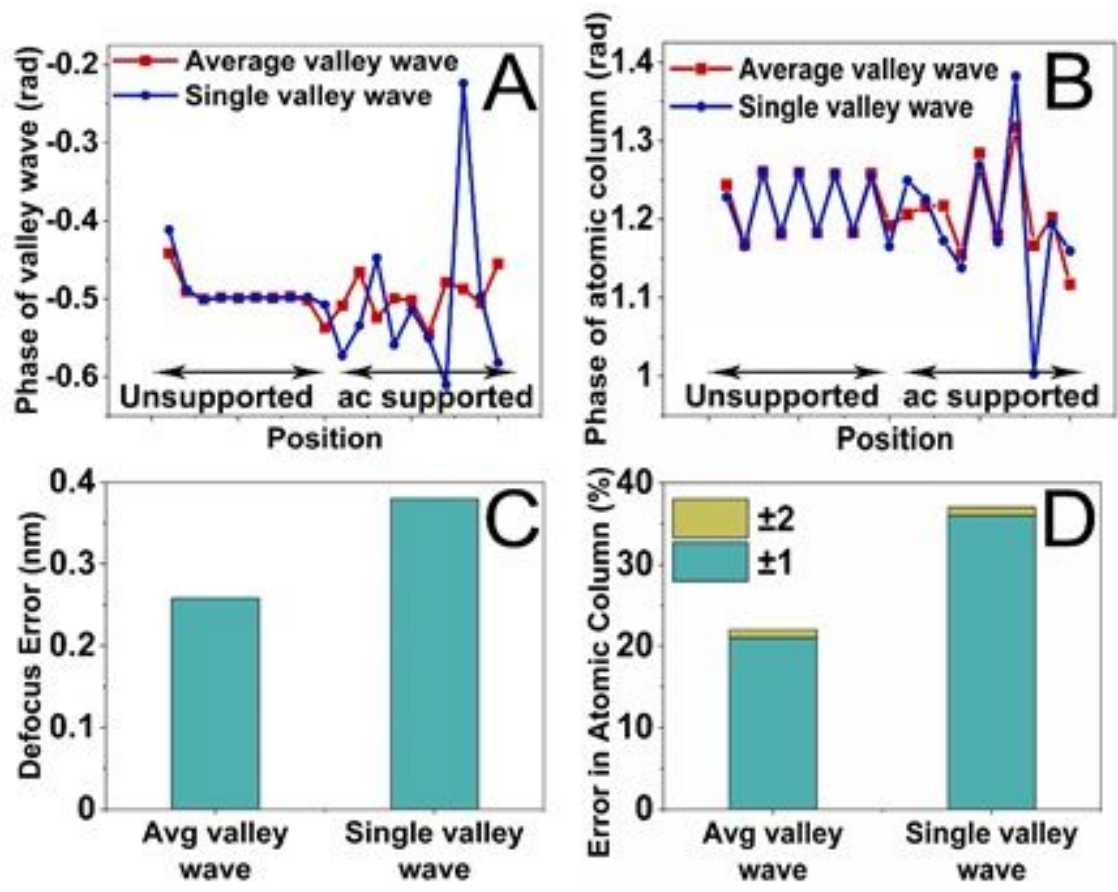

\section{Figure 2}

Effect of valley wave selection on 3D reconstruction of simulated Ag nanoparticle (A) and (B) represent the effect of valley wave selection on phase of valley wave surround the atomic column and atomic columns (FCW) respectively, atomic columns selected for line profile is shown in phase of $\mathrm{Ag}$ nanoparticle in row 3 column 2 of Fig. 1. (C) and (D) Focus and mass thickness error respectively due to valley wave selection of simulated Ag nanoparticle supporting on amorphous carbon film having $\mathrm{tp} / \mathrm{tac}$ $=1$, shown in row 3 column 1 of Fig. 1 . Colour bar in mass thickness error represent the error in number of atoms. 

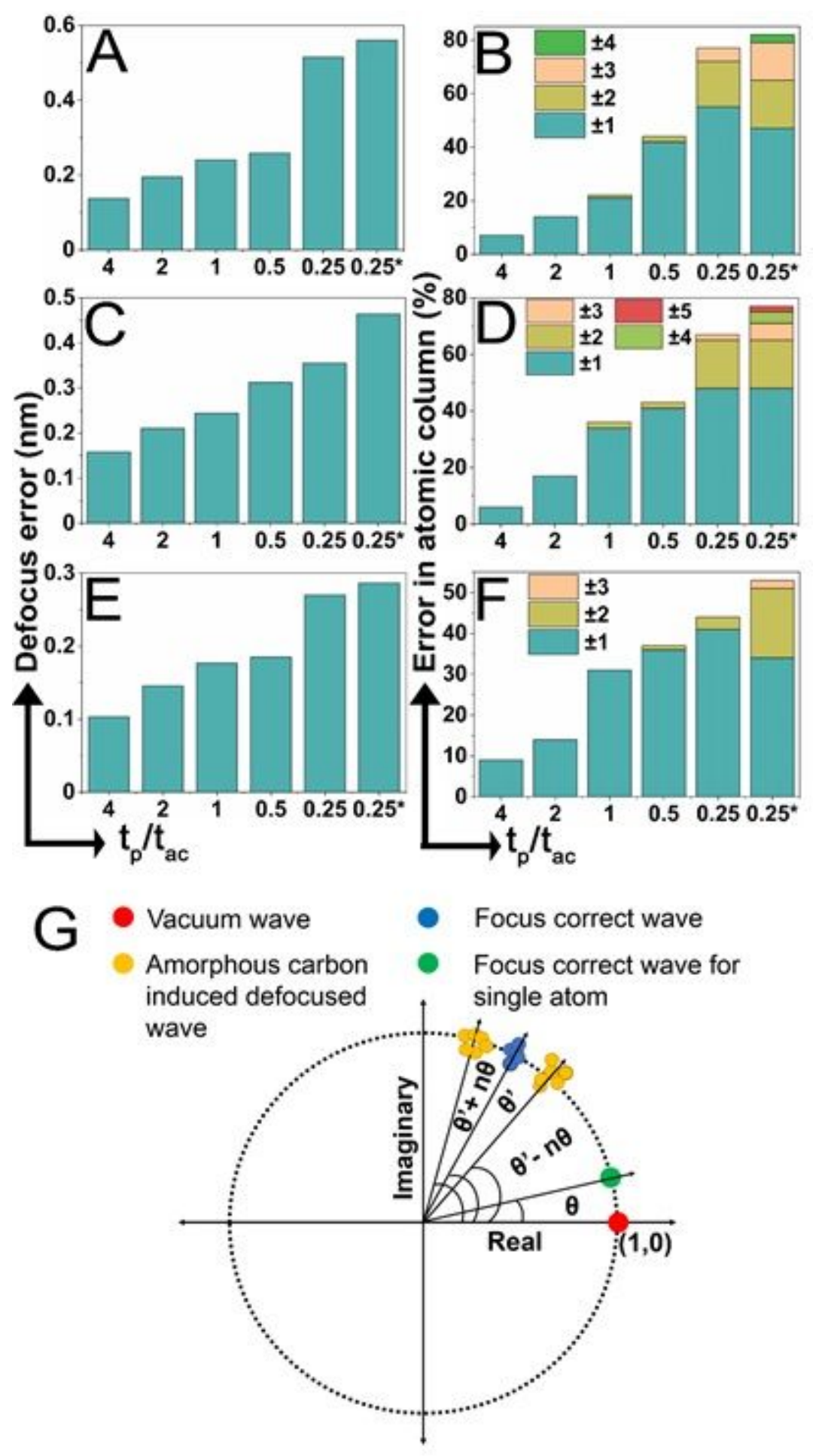

Figure 3

Representation of error in 3D reconstruction with respect to tp/tac ratio $\left(4,2,1,0.5,0.25\right.$ and $\left.0.25^{\star}\right)$ and amorphous carbon density ( $2.1 \mathrm{~g} / \mathrm{cc}$ and $3 \mathrm{~g} / \mathrm{cc}$ ) of the supporting film. (A), (C) and (E) Focus error due to varying tp/tac ratio and support carbon density for simulated $\mathrm{Ag}, \mathrm{Cu}$ and Au nanoparticle respectively. (B), (D) and (F) Mass thickness error due to varying tp/tac ratio and support carbon density for simulated $\mathrm{Ag}, \mathrm{Cu}$ and $\mathrm{Au}$ nanoparticle respectively. Colour bar in mass thickness error represent the error in atom counts. (G) Schematic showing the effect of amorphous carbon support on mass circle in Argand plane 
for disc shaped structure. Bar related to amorphous carbon density of $3 \mathrm{~g} / \mathrm{cc}$ and $\mathrm{tp} / \mathrm{tac}=0.25$ are marked with "*" sign in the figures.

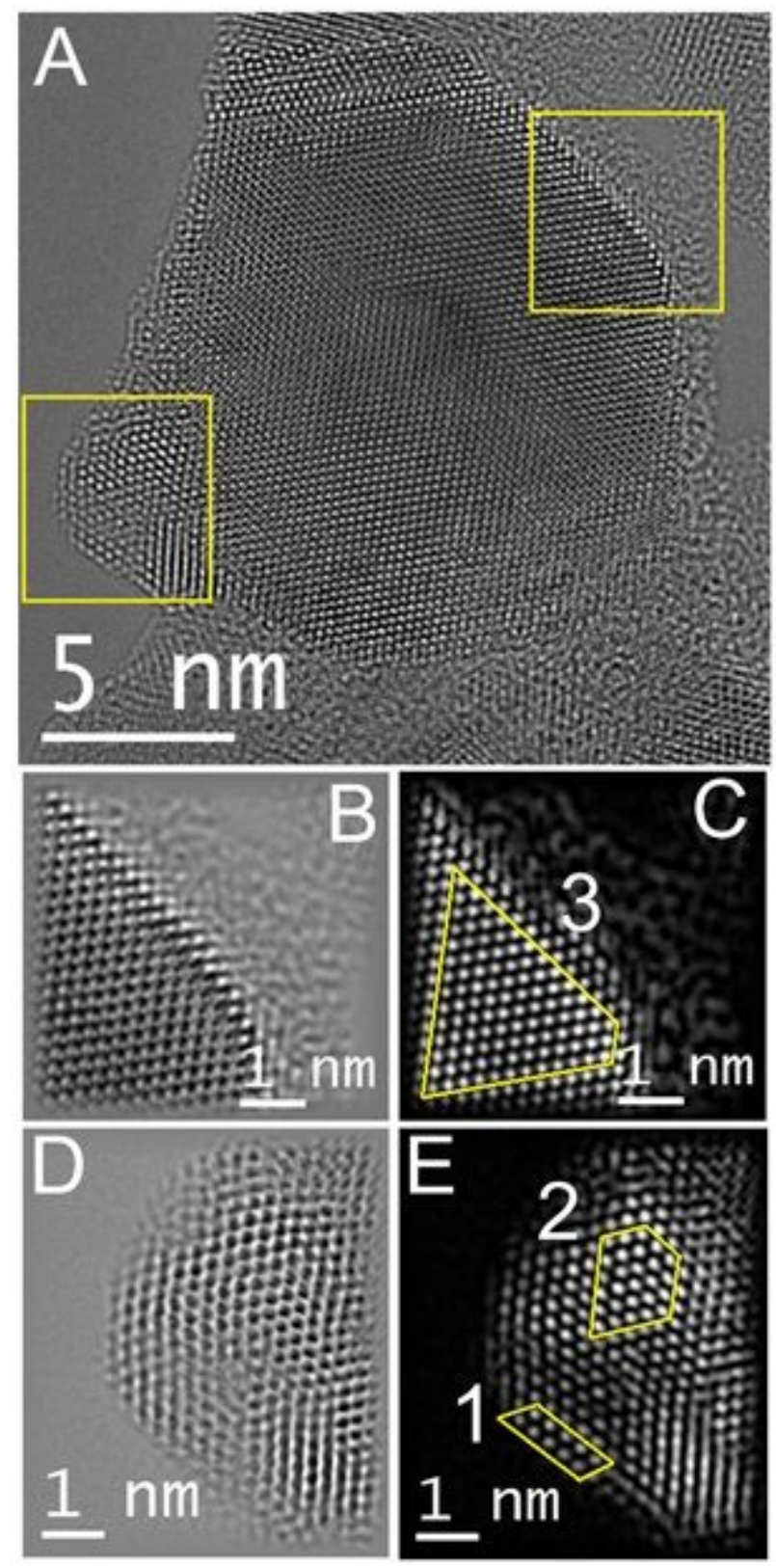

Figure 4

In-line holography of Ag nanoparticle. (A) One experimental high resolution lattice image from set of focal series containing Ag nanoparticle supported on holey carbon film viewed along $\langle 110\rangle$ direction.

Reconstructed amplitude (B) and phase (C) of the complex valued exit wave of the rectangular region marked in right upper portion in image presented in (A). Reconstructed amplitude (D) and phase (E) of the complex valued exit wave of the rectangular region marked in left lower portion in image presented in (A). Region 1 and 2 marked in (E) and 3 marked in (C) were selected further for 3D reconstruction as they have different $\mathrm{tp} / \mathrm{tac}$ ratios. 

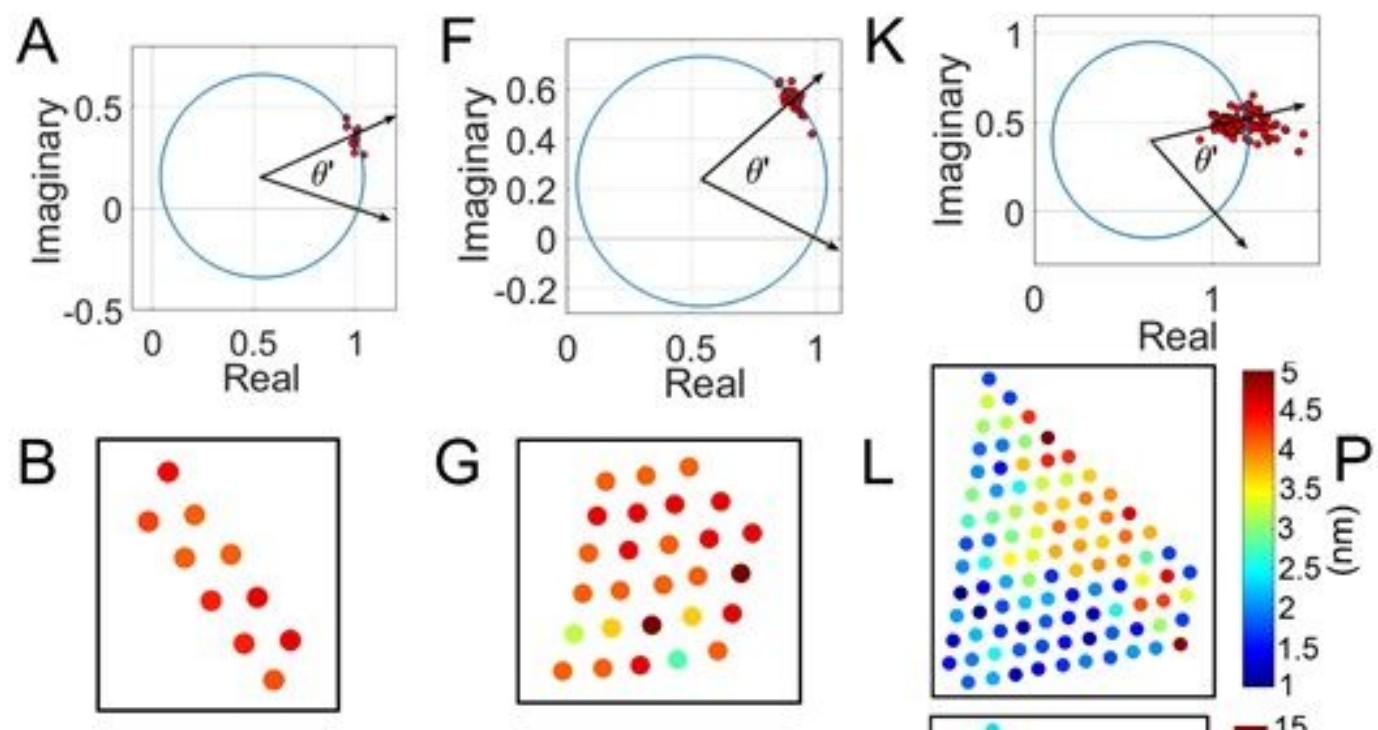

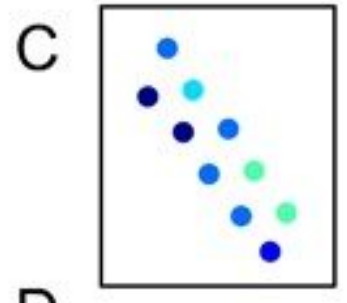

$\mathrm{D}$
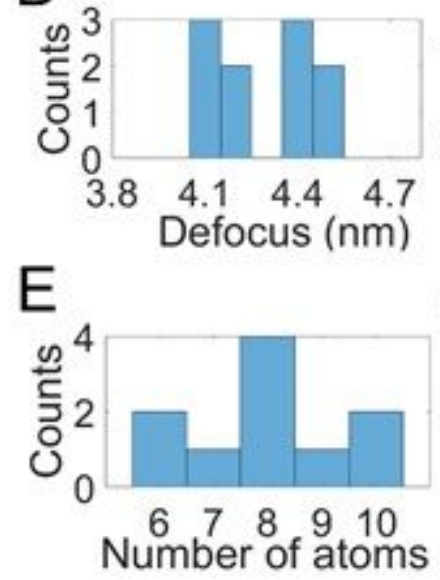
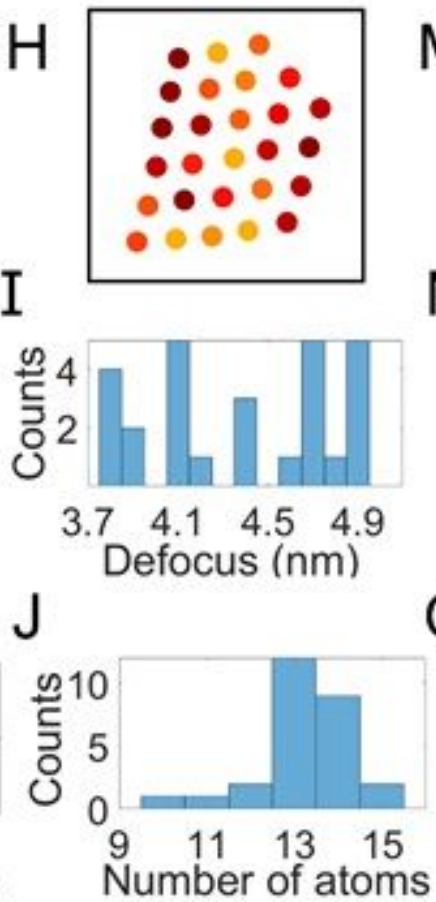
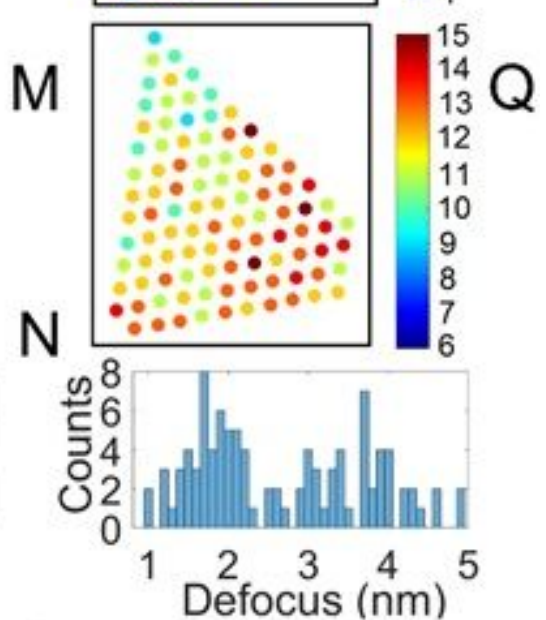

0

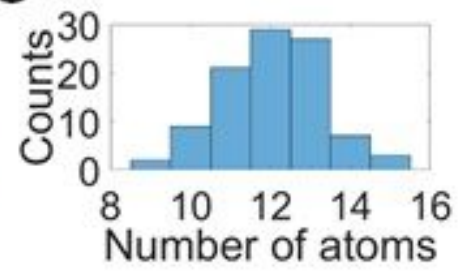

Figure 5

$3 \mathrm{D}$ reconstruction of experimental Ag nanoparticle with varying tp/tac ratio. (A-E) represent plot of the mass circle and phase $\theta^{\prime}$ in Argand plot, focus map, mass thickness map, histogram of focus values from focus map and number of atoms from mass thickness map respectively of region 1 marked in Fig. 1 (E). Similarly, (F-J) and (K-O) represent the results related to region 2 marked in Fig. 1 (E) and region 3 marked in Fig. 1 (C) respectively. In Argand plots shown in (A), (F) and (K), red dots represent FCWs and points $(1,0)$ represent vacuum waves related to respective regions. $(P)$ Common colour bar for focus map of region 1, 2 and 3. (Q) Common colour bar for mass thickness map of region 1, 2 and 3. 


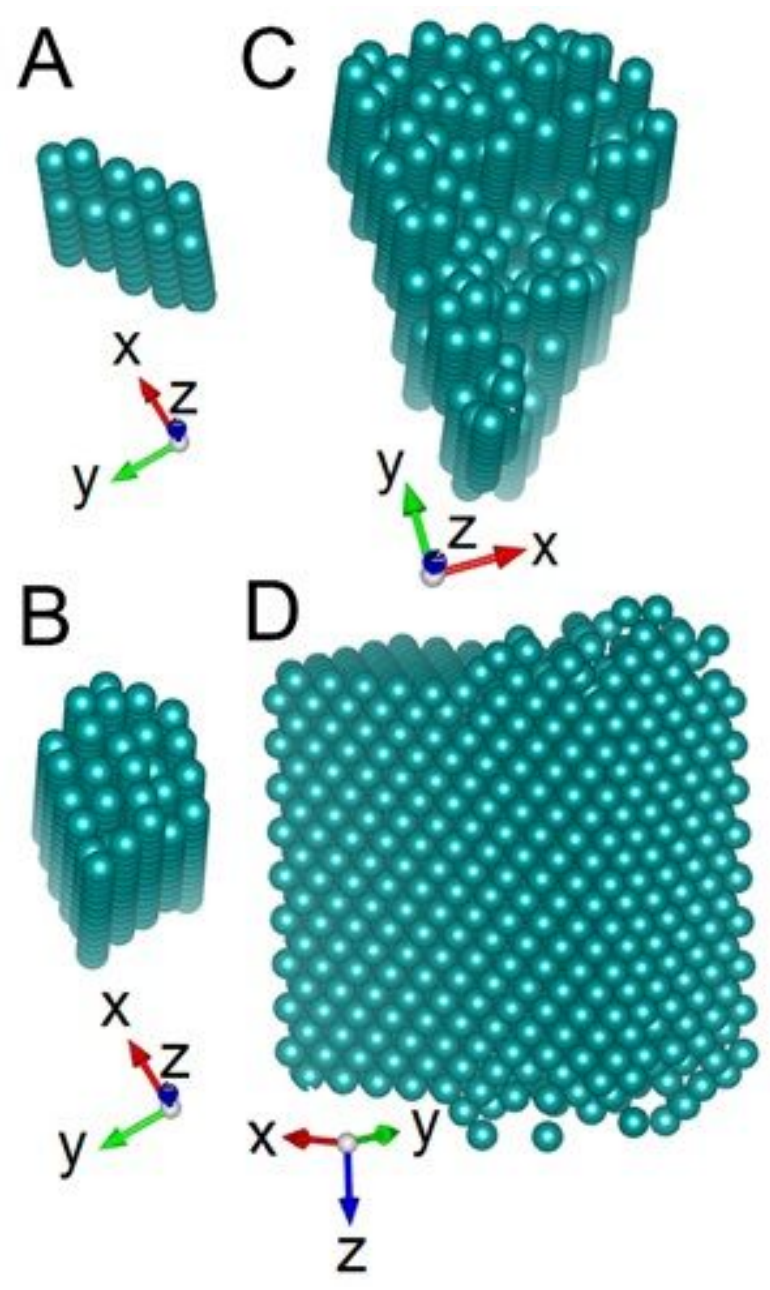

Figure 6

Atomically resolved tomograms of experimental and simulated Ag nanoparticles from single projection. (A - C) Tomogram of experimental Ag nanoparticles of region 1, 2 and 3 along [110] direction, where, $x=$ [-111], $y=$ [1-12], $z=$ [110]. (D) Tomogram of simulated Ag nanoparticle of tp/tac ratio $=1$ along [001] direction, where $x=[100], y=[010]$ and $z=[001]$.

\section{Supplementary Files}

This is a list of supplementary files associated with this preprint. Click to download.

- Supplimentaryacsnano.docx 